1 Universidade de São Paulo (USP) - São Paulo (SP), Brasil.

lytuchimura@gmail.com

2 Universidade de São Paulo (USP) - São Paulo (SP), Brasil.

analuizaviana@usp.br

3 École de Santé Publique de I'Université de Montréal - Montreal (Quebec),

Canadá.

hudsonpacifico@gmail.com

4 Faculdade de Ciências Médicas da Santa Casa de São Paulo - São Paulo (SP), Brasil.

nelson.ibanez@butantan. gov.br

\section{Unidades de Pronto Atendimento (UPAs): características da gestão às redes de atenção no Paraná}

\author{
Emergency Care Units (UPAs): management features to the care \\ networks in Paraná
}

Liza Yurie Teruya Uchimura', Ana Luiza d'Ávila Viana², Hudson Pacífico da Silva³, Nelson Ibañez ${ }^{\mathbf{4}}$

RESUMO As Unidades de Pronto Atendimento (UPAs) são classificadas, segundo a Política Nacional de Atenção às Urgências, de acordo com a capacidade física instalada, o número de leitos disponíveis, gestão de pessoas e a capacidade diária de realizar os atendimentos médicos. Objetivou-se identificar as características estruturais e de planejamento do serviço das UPAs às redes de atenção no Paraná. É um estudo de casos com entrevistas semiestruturadas com os coordenadores das UPAs, realizadas em 2012. Os dados foram armazenados e analisados em frequências absolutas e relativas. As UPAs necessitam estar integradas em uma política de Redes de Atenção à Saúde para ampliar a sua resolutividade.

PALAVRAS-CHAVE Atenção secundária à saúde; Serviços de saúde; Emergências.

ABSTRACT The Emergency Care Units (UPAs) are classified from the National Policy of Emergency Care, according to the installed physical capacity, the number of beds available, people management, and the ability to perform daily medical consultations. This study aimed to identify the structural characteristics and of service planning of the UPAs of the Parana care networks. It is a case study with semi-structured interviews carried out with UPAs coordinators, in 2012. The data were stored and analyzed in absolute and relative frequencies. The UPAs need to be integrated into a health care network policy to expand its resoluteness.

KEYWORDS Secondary Care; Health services; Emergencies. 


\section{Introdução}

O comprometimento social com a melhoria do atendimento de urgências e emergências de populações e comunidades proposto pelo sistema de saúde do Brasil enfrenta desafios decorrentes do envelhecimento da população, aumento da morbidade de causas externas, crescente incorporação tecnológica e aumento da demanda por serviços de saúde (RYCHETNIK, 2004). Em 2003, o Ministério da Saúde instituiu a Política Nacional de Atenção às Urgências (PNAU) com o objetivo de garantir a universalidade, a equidade e a integralidade no atendimento às urgências. A PNAU foi reformulada em 2011, com a instituição da Rede de Atenção às Urgências no Sistema Único de Saúde (SUS), com a finalidade de articular e integrar todos os equipamentos de saúde. As Unidades de Pronto Atendimento (UPAs) e o conjunto de serviços de urgência 24 horas são componentes da rede, que deve ser implementada gradativamente em todo território nacional com base em critérios epidemiológicos e de densidade populacional (IBAÑEZ, 2013A).

Segundo Dourado (2013), a construção da rede de urgências e emergências no cenário nacional pode ser delimitada em três períodos: o primeiro, com predomínio $\mathrm{da}$ implementação de Sistemas Estaduais de Referência Hospitalar para o atendimento de Urgências e Emergências (priorização do Serviço de Atendimento Móvel de Urgência - Samu como estratégia); o segundo, focado na implantação das UPAs; e o terceiro, insere a rede de urgências e emergências em uma rede de atenção, conforme a política nacional de conformação de redes.

As Redes de Atenção à Saúde (RAS), estabelecidas em 2010, são arranjos organizativos de ações e serviços de saúde, de diferentes densidades tecnológicas, que, integradas por meio de sistemas de apoio técnico, logístico e de gestão, buscam garantir a integralidade do cuidado, destacando que a atenção às urgências deve estar organizada dessa forma. As redes são uma garantia do direito à saúde por intermédio da ampliação do acesso, amenizando as desigualdades SOciais (KUSCHNIR; CHORNY, 2010).

Diretrizes das UPAs foram estabelecidas para a implantação no País:

Art. 2 - Definir como Unidade de Pronto Atendimento (UPA) o estabelecimento de saúde de complexidade intermediária entre as Unidades Básicas de Saúde (UBS)/Saúde da Família e a Rede Hospitalar, associada a uma rede organizada de atenção às urgências. (BRASIL, 2009, P. 33).

De acordo com o porte das UPAs, foram estabelecidas normativas para a capacidade física instalada, o número de leitos disponíveis, gestão de pessoas e a capacidade diária de realizar os atendimentos médicos. Em junho de 2011, foi publicado o Decreto Federal $n^{0} 7.508$, com o importante papel de regulamentar a Lei $n^{\circ} 8.080$ nas questões relativas a: organização do SUS, planejamento da saúde, assistência à saúde e articulação interfederativa. Sequencialmente, a PNAU foi reformulada com a criação da Rede de Atenção às Urgências no SUS. O documento definiu as diretrizes da rede, sua estrutura hierarquizada, descrevendo de forma pormenorizada seus componentes e os objetivos a serem alcançados com a regionalização dos atendimentos (BRASIL, 2011).

As UPAs foram criadas para atuar como porta de entrada aos serviços de urgência e emergência; e dependendo do caso atendido, eles poderiam ser solucionados no local, estabilizados e/ou encaminhados para os hospitais ou redirecionados às UBS. De acordo com as suas atribuições, as UPAs deveriam funcionar 24 horas por dia, realizar a classificação de risco dos pacientes, resolver os casos de baixa e média complexidade, estabilizar os casos de pacientes graves e possuir estrutura física, recursos humanos e tecnológico suficientes para o atendimento da população de sua área de abrangência (SILVA ET AL., 2012).

Passados mais de dez anos da instituição da PNAU, evidências sugerem que os esforços 
para sua implementação ainda não foram suficientes para resolver um grande contingente de problemas na saúde, como a superlotação dos serviços e atendimento de condições sensíveis à atenção primária (IBAÑEZ, 2013A).

O presente artigo utiliza parte dos dados da pesquisa multicêntrica "Rede de atenção às urgências e emergências: avaliação da implantação e desempenho das Unidades de Pronto Atendimento (UPA)" (IBAÑEZ, 2013B, P. 122), sob a coordenação do Centro de Estudos Augusto Leopoldo Ayrosa Galvão (Cealag) da Faculdade de Ciências Médicas da Santa Casa de São Paulo, e tem como objetivo central identificar as características das UPAs e a sua inserção nas Redes de Atenção às Urgências e Emergências (RUE) no estado do Paraná.

A escolha do Paraná para este estudo está justificada por ser um dos estados que concentrava $80 \%$ das UPAs em 2012 no Brasil e por apresentar um contexto estadual - do ponto de vista histórico-estrutural (com um sistema de urgência e emergência incipiente na década de 1990), político-institucional (apresentar um processo de regionalização em ampla discussão no estado) e conjuntural (atuação do Serviço Integrado de Atendimento ao Trauma em Emergência - Siate e Samu com ampla abrangência estadual) - favorável à implantação das UPAs.

\section{Métodos}

Esta pesquisa realizou um estudo de casos do processo de construção da RUE no Paraná, por meio de entrevistas semiestruturadas com os coordenadores das UPAs desse estado. Foram utilizados como dados secundários desta pesquisa o Plano Estadual de Saúde do Paraná 2012-2015 (PARANÁ, 2013) e o Cadastro Nacional de Estabelecimentos de Saúde (CNES).

Em 2010, o Paraná possuía 399 municípios e uma população de 10.444 .526 habitantes. Curitiba, Londrina, Maringá, Ponta Grossa, Cascavel, São José dos Pinhais, Foz do Iguaçu e Colombo são os municípios com populações superiores a 200 mil habitantes. Atualmente, o estado apresenta 4 Regiões Metropolitanas (RM): Curitiba (29 municípios), Maringá (25 municípios), Umuarama (23 municípios) e Londrina (18 municípios).

No ano de 2012, o estado do Paraná contava com 14 UPAs segundo o CNES, e para a pesquisa, foram selecionados 7 serviços, abrangendo $50 \%$ do universo mesmo com uma amostra intencional. Foram estabelecidos como critérios de inclusão da amostra: localização, porte e abrangência regional das UPAs. Estas deveriam representar a região metropolitana (4 UPAs) ou do interior (3 UPAs), acrescentando-se que os serviços deveriam representar diferentes municípios e regionais de saúde. A amostra ficou constituída de UPAs situadas em 6 municípios do Paraná - Curitiba (2 UPAs), Castro (1), Foz do Iguaçu (1), Cascavel (1), Maringá (1) e Sarandi (1) - e 5 regionais de saúde $-2^{\text {a }}$ regional (2 UPAs), $3^{\text {a }}$ regional (1), $9^{\mathrm{a}}$ regional (1), $10^{\mathrm{a}}$ regional (1) e $15^{\mathrm{a}}$ regional (2). Quanto ao porte, apresentaram porte I (1 UPA), porte II (2 UPAs) e porte III (4 UPAs). Foram excluídas da amostra as UPAs com menos de 6 meses de funcionamento.

As entrevistas semiestruturadas foram previamente agendadas e realizadas pelo pesquisador com todos os coordenadores das UPAs no período de dezembro de 2012 a março de 2013. O questionário foi realizado com perguntas abertas e fechadas, e o entrevistado poderia discorrer sobre o tema proposto. Foram analisadas as informações sobre o porte da unidade, população de abrangência atendida, serviços de apoio diagnóstico disponíveis, origem dos pacientes atendidos nas UPAs, relação entre a demanda e a capacidade de atendimento, frequência dos atendimentos pediátricos, capacidade de resolução dos serviços, faixa etária dos pacientes com maior taxa de atendimento, frequência dos pacientes encaminhados pela Atenção Básica (AB) e Samu, serviços de referenciamento disponíveis, forma de referenciamento para a $\mathrm{AB}$ e serviços ambulatoriais e hospitalares. 
Os dados foram armazenados em planilha eletrônica no formato Microsoft Excel e posteriormente analisados no Stata 11. Sequencialmente, foi realizada a análise estatística dos dados descritivos, apresentados na forma de frequências absolutas e relativas.

O estudo foi apreciado pelo Comitê de Ética em Pesquisa com Seres Humanos da Faculdade de Medicina da Universidade de São Paulo, conforme a Resolução 196/96-CNS, e teve parecer favorável sob o $\mathrm{n}^{\mathrm{o}} 303.421 / 2011$. O Termo de Consentimento Livre e Esclarecido (TCLE) foi apresentado a todos os profissionais antes da entrevista, o qual foi formalizado com a assinatura, posteriormente à apresentação e descrição dos objetivos da pesquisa.

\section{Resultados}

\section{Contexto das redes de urgência e emergência no Paraná}

No Paraná, a Rede de Atenção às Urgências (RAU) foi implantada em 1998 com a regulação médica de urgência e de um sistema de transporte inter-hospitalar de pacientes. Anteriormente, o sistema de urgência e emergência era composto apenas por hospitais filantrópicos e pelo Hospital da Universidade Federal do Paraná (UFPR). Apesar disso, a RAU obteve recursos orçamentários e uma organização administrativa somente a partir de janeiro de 2011, com a implantação da Rede Paraná Urgência, que tinha como principal objetivo a integração macrorregional das situações de urgência e emergência do estado por meio de ações e serviços destinados ao atendimento de todas as regiões de saúde. A Rede Paraná Urgência é formada pelos seguintes componentes: promoção, prevenção e vigilância; atenção primária na saúde; Samu/Siate; atendimento aeromédico; sala de estabilização; UPAs 24 horas; hospitais/porta de urgência hospitalar; atenção domiciliar; complexo regulador; e a telemedicina com linhas de cuidados cardiovascular, cerebrovascular e trauma.

As ações da promoção, prevenção e vigilância se apresentam concentradas em serviços intersetoriais, campanhas educativas e no VigiaSUS (campanhas de vigilância e prevenção). Para a atenção primária, foi determinada a competência pelo primeiro atendimento para todos os graus de urgência, com um atendimento resolutivo para os casos de gravidade de baixo risco, implantação de kits de emergência nas UBS/Estratégia Saúde da Família (ESF) e sua integração à classificação de risco de Manchester. Por fim, o sistema de Regulação do Paraná, que é composto por sete Centrais Regionais de Leitos e Consultas (Curitiba, Londrina, Maringá, Ponta Grossa e Cascavel) e quatro Centrais Municipais.

O Paraná possui 22.420 leitos hospitalares, ofertados em 458 hospitais públicos, filantrópicos ou privados que atendem o SUS, dos quais 72,3\% têm menos de 50 leitos. Foi criado o Programa de Apoio e Qualificação de Hospitais Públicos e Filantrópicos do SUS Paraná (HOSPSUS) para qualificar a resposta hospitalar em todas as regiões de saúde, contribuindo para o desenvolvimento social e sanitário, essencial para atender às necessidades da população do estado, a partir de uma estratégia ligada à regionalização. Atualmente, existem 49 hospitais cadastrados no HOSPSUS, que possuem no mínimo 100 leitos ofertados ao SUS e que são referenciados na região para a média e alta complexidade; eles atendem no mínimo $25,0 \%$ de pacientes referenciados de sua região de abrangência e são indicados como hospitais de referência regional e/ou macrorregional (PARANÁ, 2013).

A Rede Paraná Urgência tem como diretrizes gerais: a organização da atenção primária em todos os municípios paranaenses; a implantação da classificação de risco de Manchester em todos os níveis de atenção à saúde; o estabelecimento de critérios de atendimento com associação ao tempo-resposta (principalmente para atendimentos 
cardiovascular, neurovascular e trauma); o estabelecimento de competências dos vários níveis de atenção à saúde; a definição dos atendimentos por linhas de cuidado e dos pontos regionalizados com implantação de transferência sob regulação; implementação de pactuações interestaduais; e implantação de Programa de Educação Permanente em Atenção às Urgências às equipes de saúde (SCARPELINI, 2007).

Desde 1990, o Sistema Integrado de Atendimento ao Trauma e Emergências (Siate) funciona no Paraná por intermédio da parceria com o Corpo de Bombeiros, Secretaria Estadual de Saúde (Sesa) e municípios, com atendimento especializado ao trauma e regulação médica. A partir de 1995, o Siate se expandiu para o interior do estado e atualmente está implantado pelo modelo integral em Curitiba, São José dos Pinhais, Campo Largo, Ponta Grossa, Londrina, Maringá, Foz do Iguaçu e Cascavel. Além desses municípios, o Siate realiza cobertura em outras sete cidades de maneira parcial, ou seja, sem regulação e intervenção médica próprias. Semelhantemente ao que ocorre em muitos municípios brasileiros, o Siate Paraná possui um sistema misto de urgência e emergência, ou seja, utiliza ambulâncias equipadas para o atendimento com socorristas como o modelo americano ou com médicos de acordo com o modelo francês (SCARPELINI, 2007).

O Samu foi implantado inicialmente, em 2004, em Curitiba, e até o final de 2011 estava presente em 15 municípios, tendo como população de abrangência $44,0 \%$ dos habitantes do estado. O PDR 2012-2015 propõe que, até o fim desse período, $100,0 \%$ da população seja atendida pelo Samu e que $50,0 \%$ das internações de urgência e emergência sejam reguladas pela Central de Regulação (PARANÁ, 2013).

\section{Caracterização geral das UPAs}

As UPAs estavam localizadas na região metropolitana de Curitiba (Pinheirinho e Sítio Cercado), na região metropolitana de Maringá (Zona Sul e Sarandi), em Foz do Iguaçu (João Samek), em Cascavel (Tancredo Neves) e em Castro (Moacir Elias Fadel). Segundo o modelo de gestão atuante nas UPAs, todas referiram possuir administração direta, ou seja, por meio das respectivas Secretarias Municipais de Saúde.

A UPA Sítio Cercado, em Curitiba, é a mais antiga e foi implantada em 2000; era originalmente o Centro Municipal de Urgências Médicas (CMUM) e foi transformada em UPA em 2012. Da mesma forma, a UPA Pinheirinho, também em Curitiba, foi inaugurada como CMUM em 2006 e transformada em UPA em 2012. As UPAs de Curitiba foram implantadas anteriormente à política nacional, seguindo os critérios populacionais, fluxo de pacientes preestabelecidos e condições técnicas para implantação do serviço; desde então, possuíam o objetivo de dar atendimento a casos de média complexidade. Nesse período, o gerenciamento dos CMUMs era realizado por contratos com hospitais do município. A mudança para UPAs já havia sido discutida em outras gestões, mas sempre houve resistência devido à afirmação da identidade local em anos anteriores. A fim de modificar esse panorama e seguir o proposto pelo Ministério da Saúde, a partir de 2012, o município de Curitiba padronizou suas diretrizes de acordo com a Política Nacional de Urgência e Emergência.

A UPA Tancredo Neves, em Cascavel, criada em 2002, foi inaugurada como Pronto Atendimento Continuado (PAC), sendo posteriormente transformada em UPA em 2011. O processo de transformação do PAC de Cascavel em UPA ocorreu em consequência de negociações entre a promotoria geral, gestores estaduais e municipais, para descentralizar os atendimentos de urgência e emergência da macrorregião de Cascavel para o Pronto-Socorro do Hospital Universitário da Universidade Estadual do Oeste do Paraná (Unioeste).

Segundo o gestor municipal da UPA de Castro, a implantação do serviço foi 
justificada apenas na questão da organização da RAU da região. Até o momento da realização da pesquisa, em dezembro de 2012, Castro era o único município da $3^{\text {a }}$ Região de Saúde que possuía uma UPA funcionando para atendimentos. Com uma população de 67 mil habitantes, esse município, de acordo com a classificação da Portaria $\mathrm{n}^{\mathrm{o}}$ 1.601, necessitaria apenas de uma UPA de porte I; entretanto, por intervenção da Secretaria Estadual de Saúde, foi implantado um serviço de maior capacidade, a fim de ampliar os atendimentos para os municípios próximos a Castro.

As UPAs Zona Sul (Maringá), Sarandi, Moacir Elias (Castro) e a João Samek (Foz do Iguaçu) foram inauguradas, a partir do ano de 2010, após a portaria federal de criação dos serviços de UPA.

As justificativas referidas para a implantação das UPAs no Paraná foram diversas: para quatro delas (57,1\%), estariam ligadas a condições socioeconômicas, ao tamanho e à concentração de população na área; 71,4\% referiram insuficiência de serviços de saúde; $85,7 \%$ que a implantação estava relacionada à organização da RAU na região; $42,8 \%$ pela reivindicação da população/comunidade; $57,1 \%$ pela atuação de políticos/parlamentares; $14,3 \%$ revelaram que a implantação no território estaria vinculada aos indicadores de violência e mortalidade.
Com relação à disponibilidade dos cursos de capacitação em urgência/emergência oferecidos aos médicos que atuam nas UPAs, $4(57,1 \%)$ dos entrevistados referiram que não há cursos disponíveis para os médicos. A capacitação voltada aos enfermeiros que trabalham nesses serviços possui as mesmas frequências que os cursos para os médicos. Para os demais profissionais de saúde, a disponibilidade dos cursos de capacitação foi referida por $57,1 \%$; entre estes profissionais, constam: auxiliar de serviços gerais, administrador e técnico de enfermagem.

Entre os Serviços de Apoio Diagnóstico e Terapêutico (SADT) oferecidos nas UPAs, constam: o serviço de radiologia em $85,7 \%$; eletrocardiograma em $100 \%$; laboratório de análises clínicas em 85,7\%; ultrassonografia em $28,8 \%$; ecocardiografia e endoscopia em $14,3 \%$.

Com relação à capacidade de resolução dos problemas de saúde atendidos, $57,1 \%$ dos coordenadores das UPAs a avaliam como adequada, para $28,57 \%$ foi avaliada como razoável e 14,28 \% não souberam informar a capacidade de resolução de seu serviço.

As avaliações das UPAs eram realizadas de várias maneiras, sendo 14,3\% pelo Ministério da Saúde ou Secretaria do Estado de Saúde; 28,6\% pela Secretaria Municipal de Saúde e $42,9 \%$ relataram que as avaliações eram realizadas pelo Conselho de Saúde (quadro 1).

Quadro 1. Características da amostra de UPAs no Paraná, 2013

\begin{tabular}{llllll}
\hline \multicolumn{1}{c}{ UPAs } & \multicolumn{1}{c}{ Município } & $\begin{array}{c}\text { Região de } \\
\text { Saúde }\end{array}$ & Localização & Porte & Gestão \\
\hline Pinheirinho & Curitiba & 20 & Metropolitana & II & Municipal \\
Sítio Cercado & Curitiba & 20 & Metropolitana & III & Municipal \\
Zona Sul & Maringá & 150 & Metropolitana & III & Municipal \\
Sarandi & Sarandi & 150 & Metropolitana & I & Municipal \\
João Samek & Foz do lguaçu & 90 & Interior & III & Municipal \\
Tancredo Neves & Cascavel & 100 & Interior & III & Municipal \\
Moacir Elias Fadel & Castro & 30 & Interior & II & Municipal \\
\hline
\end{tabular}

Fonte: PARANÁ, 2013 
Em três UPAs, os mecanismos de avaliação estavam implantados no serviço, sendo $57,1 \%$ por intermédio de reuniões com os gestores do SUS (Ministério da Saúde, Secretaria Municipal e/ou Estadual); 71,4\% por meio da supervisão de atividades do Conselho de Saúde (CS) e/ou Secretaria
Municipal de Saúde (SMS) e 57,1\% por meio de relatórios de gestão. Essas avaliações ocorrem em 14,3\% dos serviços de maneira mensal ou quadrimestral, ou anual, ou com todas estas frequências associadas, enquanto $42,8 \%$ não souberam informar a periodicidade da avaliação (quadro 2) .

Quadro 2. Sistema de gerenciamento das UPAs. Paraná, 2013

\begin{tabular}{|c|c|c|c|c|}
\hline UPAs & Modelo Gestão & Avaliações & $\begin{array}{l}\text { Mecanismos de } \\
\text { avaliação }\end{array}$ & Periodicidade \\
\hline Pinheirinho & Municipal & Sesa + SMS + CS & Supervisão & Mensal \\
\hline Sítio Cercado & Municipal & - & - & Não informou \\
\hline Maringá & Municipal & $\begin{array}{l}\text { Pouco tempo de } \\
\text { funcionamento }\end{array}$ & Relatórios de gestão & Anual \\
\hline Sarandi & Municipal & SMS + CS + equipe & $\begin{array}{l}\text { Reuniões de gestores+ } \\
\text { Supervisão pelo CS + } \\
\text { Relatórios de gestão }\end{array}$ & Semanal + Mensal + Anual \\
\hline Foz do Iguaçu & Municipal & $M S+C S$ & - & Quadrimestral \\
\hline Cascavel & Municipal & - & - & Não informou \\
\hline Castro & Municipal & - & - & Não informou \\
\hline
\end{tabular}

Fonte: PARANÁ, 2013

A partir das informações dos gestores entrevistados, no conjunto das UPAs da amostra, a origem dos pacientes atendidos nos serviços se divide em: $71,2 \%$ com referência de todo o município e $28,8 \%$ com referência de uma região dentro do município (situação identificada nas unidades de Curitiba). Em relação à demanda e à capacidade de atendimento das UPAs, foi referido por seis dos serviços (85,7\% da amostra) que a demanda é superior à capacidade de atendimento; apenas um serviço foi referido que a demanda é compatível com a capacidade de atendimento.

Segundo a Portaria $n^{0} 1.601$, de julho de 2011, que estabeleceu diretrizes para implantação das UPAs, os atendimentos pediátricos também devem ser preconizados de maneira regular, porém, em 14,3\% dos serviços, estes atendimentos são realizados de forma esporádica, diferentemente dos outros serviços que ocorrem com frequência. Observou-se ainda que, em 42,9\% das UPAs, a faixa etária de maior demanda dos pacientes atendidos eram de 20 a 39 anos.

\section{Integração das UPAs com os com- ponentes da Rede de Atenção às Urgências}

De acordo com os coordenadores, em $85,7 \%$ das UPAs está disponível o referenciamento de pacientes atendidos no serviço para as unidades de saúde da $\mathrm{AB}$ e unidades de saúde com ESF. O encaminhamento de pacientes das unidades de saúde para as UPAs está disponível em todos os serviços. Em 57,1\% dos serviços existe a disponibilidade para encaminhamento aos ambulatórios de especialidades médicas e em $42,8 \%$ aos ambulatórios de especialidades não médicas, como a fisioterapia, odontologia e 
psicologia. Para pacientes com remoção necessária, há disponibilidade em $42,8 \%$ dos serviços de encaminhamento para outras unidades de pronto atendimento e para os SADT em $57,1 \%$ dos serviços. Em $57,1 \%$ das UPAs há estabilização e atendimento precoce de pacientes transportados pelo Samu e em $42,8 \%$ os pacientes são frequentemente encaminhados pelo serviço de atendimento móvel. Para os atendimentos mais graves, os serviços de emergências hospitalares ficam disponíveis para o

Figura 1. Percentual de disponibilidade de referenciamento/contrarreferenciamento das UPAs à atenção primária, secundária e terciária segundo coordenadores das UPAs. Paraná, 2013

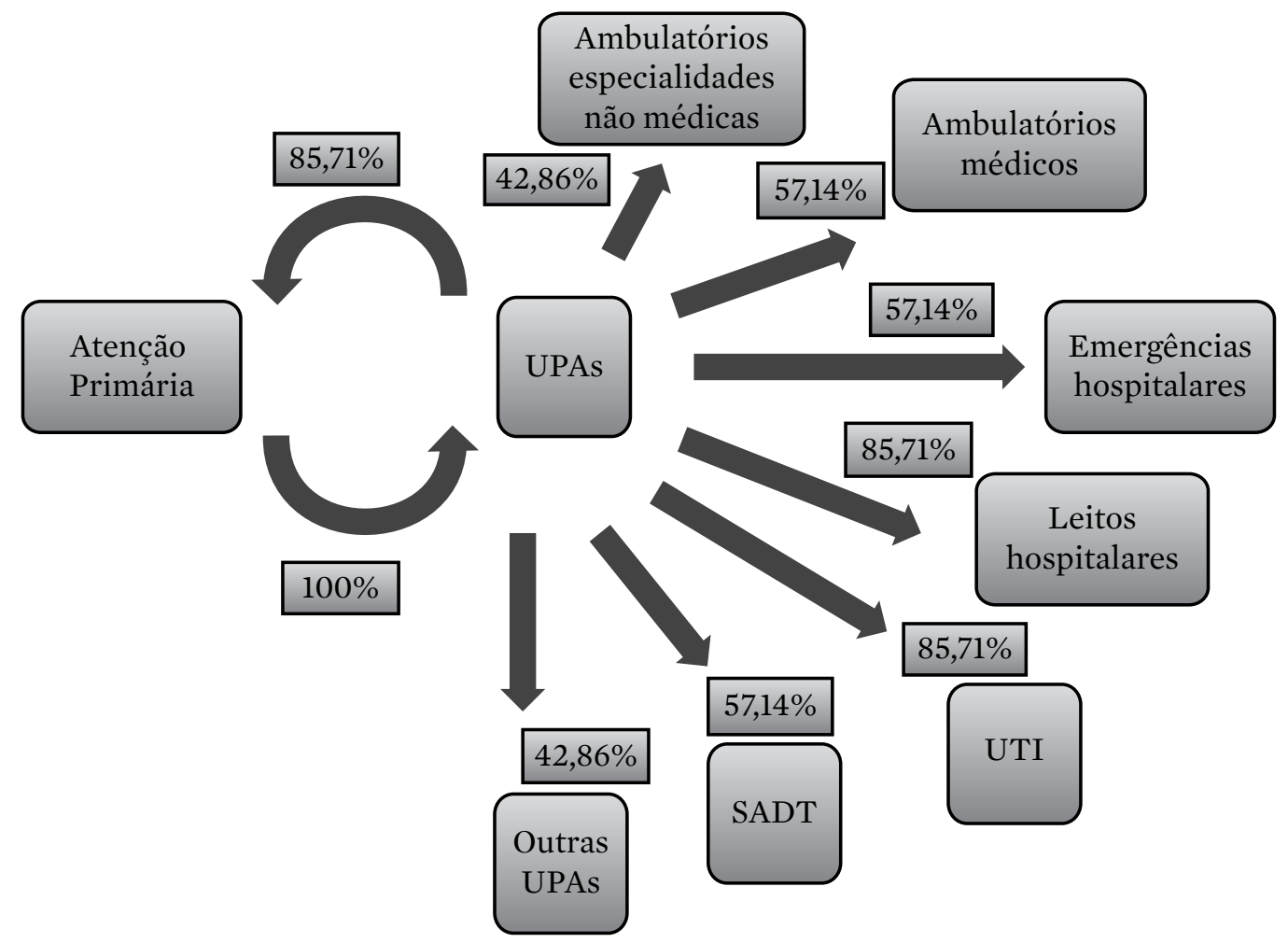

Fonte: PARANÁ, 2013.

referenciamento em $57,1 \%$ das UPAs, enquanto para leitos hospitalares e Unidades de Terapia Intensiva (UTIs) em $85,7 \%$ dos casos (figura 1).

Em todos os serviços da atenção primária e nas UPAs de Curitiba, existe um sistema eletrônico (E-saúde), desde 2000, que interliga as unidades e propicia acesso aos prontuários eletrônicos, exames de diagnóstico e relatórios de gestão e planejamento. Com essa facilidade de comunicação, é possível acessar os atendimentos a pacientes de qualquer uma dessas unidades. Este sistema funciona como instrumento de referência e contrarreferência dos componentes da RAS, mas ele depende dos profissionais de saúde para a realização do registro. O serviço de referência e contrarreferência entre a atenção hospitalar e as UPAs é realizado por meio de relatórios médicos impressos.

O serviço de referenciamento para a atenção terciária é realizado de formas diferentes: em alguns, o encaminhamento é realizado para outras cidades próximas, pois existe 
apenas um hospital particular na cidade; para outros, o referenciamento de pacientes para a $\mathrm{AB}$ é realizado por meio de contato telefônico, mas para o encaminhamento de pacientes para serviços ambulatoriais especializados, é utilizado o formulário de pacientes e a Central de Regulação municipal, destacando-se que o encaminhamento de pacientes para os hospitais é realizado por contato pessoal, central de regulação e Samu. Outros serviços referiram ainda que apesar da disponibilidade de referenciamento para os outros níveis de atenção à saúde, a referência e a contrarreferência não funcionam, no município, pela falta de adesão dos médicos aos protocolos ou a não concordância com o diagnóstico de outros profissionais.

O principal motivo para a permanência de pacientes nas UPAs por mais de 24 horas se pontuou na inexistência de vagas em todos os serviços, exceto em um, que relatou a falta de serviços para o referenciamento. Também foi relatado como causa: a deficiência no seguimento do protocolo de fluxo dos pacientes e a dificuldade de contratação de médicos no serviço. Todos os coordenadores negaram que os motivos estariam ligados à deficiência de transporte por mecanismos formais de encaminhamento.

Com relação ao retorno de pacientes atendidos previamente com as mesmas queixas, duas UPAs referiram que este procedimento é frequente, para quatro UPAs o retorno dos pacientes ocorre em alguns casos e para uma UPA este fato é raro.

\section{Discussão}

A implantação das primeiras propostas organizadas de oferta de serviços regionalizados de Urgência e Emergência em ambiente não hospitalar em Curitiba, no Paraná, ocorreram por intermédio dos CMUMs antes mesmo da normatização, pois já possuíam serviços capacitados e com funções específicas para o atendimento entre a atenção primária e a hospitalar. O'Dwyer (2010), em seu estudo, questiona a implantação das UPAs no processo decisório político, pois refere que essa estratégia seria pontuada como uma necessidade técnica ou como um impacto político eleitoral. Mesmo com a implantação prévia de três unidades para o atendimento da atenção secundária no Paraná, a distribuição dos recursos orçamentários e a formalização administrativa somente ocorreram a partir de janeiro de 2011, dando início à Política de Urgência do Estado.

O federalismo atual, ao mesmo tempo que exige responsabilidades, disponibiliza incentivos para políticas cooperativas com os estados e os municípios, porém a dependência do financiamento federal pode criar situações conflitivas, prejudicando as possíveis políticas cooperativas (O'DWYER, 2010).

Novaes (2004) destaca a importância de avaliações em saúde como um item essencial para as tomadas de decisões na gestão e a identificação dos impactos da tecnologia ou estrutura para o sistema de saúde, contribuindo para o aprimoramento do objeto avaliado. Os mecanismos de avaliações nas UPAs do Paraná estavam presentes em três serviços e eram realizados com baixa frequência.

Sobre a relação demanda e a capacidade de atendimento, nas UPAs do Paraná, $71,2 \%$ dos pacientes eram procedentes do próprio município, aumentando a demanda desses serviços e, consequentemente, caracterizando um baixo caráter regional nos atendimentos. A demanda das UPAs do Paraná era maior do que a sua capacidade, e a aparente facilidade de acesso desses serviços e a garantia de recursos (consultas, exames, medicamentos) no momento da procura são fatores considerados importantes para a população e indiretamente contribuem para um excesso de atendimentos de acordo com a sua capacidade (GOMIDE; PINTO; FIGUEIREDO, 2012). 
A constante busca pelo serviço de urgência para o atendimento da atenção primária pode demonstrar uma falha no vínculo do sistema de saúde. Assim como demonstrado no estudo de Gomide, Pinto e Figueiredo (2012) em Ribeirão Preto, São Paulo, nas UPAs do Paraná, exceto as de Curitiba, o usuário busca o seu atendimento no local onde satisfaz suas necessidades sem levar em consideração a localização geográfica do serviço. Acrescenta ainda, como fatores do aumento da demanda nas UPAs, a difícil acessibilidade organizacional nas UBS e a questão sociocultural de como o indivíduo entende o processo saúde-doença.

Konder (2013) relata que a atenção primária apresenta dificuldades em atender demandas espontâneas diante das responsabilidades preestabelecidas ou, em alguns casos, existe a falta de qualificação dos profissionais. Jimenez, Simão e Shimazaki (2003) discorrem que a capacitação profissional é considerada um eixo estratégico para ampliar a resolutividade da atenção primária e evitar que os casos não urgentes se tornem atendimentos excessivos para as UPAs. Além disso, relata que a falta de valorização do médico de família e comunidade por outros profissionais de saúde, na sociedade e na universidade, também é considerado um obstáculo para a confiança dos usuários na conduta médica.

Segundo Hartz e Contandriopoulos (2004), o princípio da integralidade do SUS se refere à integração dos serviços por meio das várias relações, a fim de proporcionar o melhor ciclo de vida aos seus usuários, determinando um 'sistema sem muros' em que as barreiras de acesso para os vários níveis de atenção são eliminadas, sendo estes apenas conectados por um eficiente sistema de informação.

Giovanella et al. (2002) relatam que o conceito da integralidade se estende também a uma oferta organizada de assistência com garantia de referência e contrarreferência na rede e acrescentam ainda que essas garantias são consideradas etapas de um estágio mais avançado de articulação entre os diversos níveis de atenção.

Em Curitiba, um elemento facilitador para esse conceito de integralidade está no sistema de prontuários do município, pois ele possibilita o acesso às informações de consultas, exames laboratoriais, uso e dispensação de medicamentos, participação de atividades de promoção de saúde e de internamentos nas unidades básicas/ESF, nas UPAs e na SMS. Além disso, é possível obter relatórios periódicos sejam eles por doenças, faixa etária dos pacientes, profissionais de saúde e área de abrangência das equipes de saúde da família, observando, no entanto, que o sistema será eficaz quando o profissional comprometido fizer o registro dos dados de forma adequada. No período da pesquisa, uma UPA estava implantando o sistema próprio de informação que integra os serviços de saúde municipais, faltando apenas a conectividade com quatro UBS e o Hospital Municipal. Acrescente-se que os serviços de referência e contrarreferência ainda não estavam estabelecidos nas UPAs dos municípios do interior do estado. Fato não específico somente dos municípios do interior, no qual foi comprovado no estudo de Almeida, Fausto e Giovanella (2011) que quatro centros urbanos em 2008 não possuíam um sistema de contrarreferência apesar de os fluxos estarem estabelecidos.

Almeida et al. (2010) afirmam que a falta de integração da rede se deve à falta de comunicação entre os diferentes prestadores, à insuficiência de fluxos preestabelecidos para a atenção terciária e à ausência de políticas para a média complexidade.

A atenção secundária com as consultas especializadas e com os atendimentos de urgências e emergências nas UPAs necessitam de reformulações em sua política nacional e na efetivação em cada município. As dificuldades na organização da atenção secundária devem ser elencadas e discutidas nos níveis federais, estaduais e municipais a fim de obterem melhores práticas em saúde.

Embora haja experiências pontuais com SUcesso (JIMENEZ; SIMÃO; SHIMAZAKI, 2003) por 
intermédio das redes temáticas no Paraná, como acontece com o Programa Mãe Curitibana, precedente do Programa Mãe Paranaense, ao pensar em RAS, o sistema se apresenta obsoleto com baixa regionalização e hierarquização dos serviços. Mesmo com um Siate e uma regulação estadual de leitos incipiente desde a década de 1990, a organização e administração dos serviços de saúde de urgência e emergência no Paraná não funcionam totalmente integradas e possuem grandes diferenças estruturais por municípios. O Programa Estadual de Apoio aos Consórcios Intermunicipais de Saúde (Comsus) propõe amenizar essas diferenças e qualificar os municípios para a atenção secundária, fortalecendo, dessa maneira, a Rede Paraná Urgência (PARANÁ, 2013).

O governo estadual do Paraná reconhece as UPAs como um componente da rede de extrema importância, no entanto, não possui mecanismos de avaliação e gestão, assim como não auxilia os municípios com os repasses oriundos da esfera federal na questão do financiamento. Dessa forma, é necessário que o estado amplie sua participação na implantação e gestão destes serviços.

\section{Referências}

\footnotetext{
ALMEIDA, P. F. et al. Desafio à coordenação dos cuidados em saúde: estratégias de integração entre níveis assistenciais em grandes centros urbanos. Cad. Saúde Publica, Rio de Janeiro, v. 26, n. 2, p. 286-298, 2010.
}

ALMEIDA, P. F.; FAUSTO, M. C. R.; GIOVANELLA, L. Fortalecimento da atenção primária à saúde: estratégia para potencializar a coordenação dos cuidados. Rev Panam Salud Publica, Washington, DC, v. 2, n. 2, p. 84-95, 2011.

BRASIL. Ministério da Saúde. Portaria n ${ }^{\circ} 1.600$ de 7 de julho de 2011. Reformula a Política Nacional de Atenção às Urgências e institui a Rede de Atenção às Urgências no Sistema Único de Saúde (SUS). Diário Oficial [da] União, Brasília, DF, 8 jul. 2011.

\section{Conclusão}

As UPAs no Paraná apresentaram diferentes problemas em cada município, evidenciando-se, entretanto, que há uma integração parcial das UPAs com as RAS, principalmente em relação à Rede Paraná Urgência. Apesar dos fluxos estarem estabelecidos pela Rede Paraná Urgência, há entraves na prática que dificultam o seguimento destes protocolos sejam eles por falta de profissionais capacitados ou pelas dificuldades estruturais de referencia/contrarreferência de pacientes na macrorregião.

As UPAs necessitam estar integradas em uma política de RAS para ampliar a sua resolutividade, com uma maior participação da esfera estadual em seu processo de implementação, revisando e reformulando os critérios de localização dos territórios. A elaboração de um sistema informatizado nas macrorregionais reduziria a demanda nos serviços e facilitaria os fluxos assistenciais. Além disso, a padronização nos mecanismos de avaliação facilitaria no processo de planejamento, e, dessa forma, as funções dos sistemas de saúde estariam presentes garantindo o direito à saúde para todos.

\footnotetext{
. Ministério da Saúde. Portaria ${ }^{\circ} 1.020$ de 13 de maio de 2009. Estabelece diretrizes para a implantação do componente pré-hospitalar fixo para a organização de redes locorregionais de atenção integral às urgências em conformidade com a Política Nacional de Atenção às Urgências. Diário oficial [da] União, Brasília, DF, 15 maio 2009.
}

DOURADO, E. M. R. Análise da Política Nacional de Atenção às Urgências: uma proposta. 2013. 154 f. Dissertação (Mestrado em Saúde Coletiva) Universidade de Brasília, Brasília, DF, 2013.

GIOVANELLA, L. et al. Sistemas municipais de saúde e a diretriz da integralidade da atenção: critérios para 
avaliação. Saúde em Debate, Rio de Janeiro, v. 26, n. 60, 37-61, 2002.

GOMIDE, M. F. S.; PINTO, I. C.; FIGUEIREDO, L.

A. Acessibilidade e demanda em uma Unidade de Pronto Atendimento: perspectiva do usuário. Acta Paul Enferm., São Paulo, v. 25, n. spe. 2, p. 19-25, 2012.

\section{HARTZ, Z. M. A.; CONTANDRIOPOULOS, A. P.}

Integralidade da atenção e integração de serviços de saúde: desafios para avaliar a implantação de um "sistema sem muros”. Cad. Saúde Pública, Rio de Janeiro, v. 20, n. 2, p. S331-S336, 2004.

IBAÑEZ, N. (Coord.). Rede de Atenção às Urgências e Emergências: avaliação da implantação e desempenho das Unidades de Pronto Atendimento (UPA). Relatório Final - Dados Gerais. São Paulo: Universidade Estadual de Campinas, set., 2013b, 122 p.

IBAÑEZ, N. Os hospitais e a Rede de Atenção às Urgências e Emergências: desafios. Rev Conselho Nacional Secretários Saúde, Brasília, DF, v. 3, n. 7, p. 39-43, 2013 a.

JIMENEZ, E. J. B.; SIMÃO, M. B. G.; SHIMAZAKI, M. E. Programa Mãe Curitibana: avançando na humanização da atenção materno-infantil. Saúde em Debate, Rio de Janeiro, n. 26, p. 21-29, 2003.

KONDER, M. T. Atenção às urgências: a integração das Unidades de Pronto Atendimento 24 horas (UPA 24h) com a rede assistencial no município do Rio de Janeiro. 2013. 108 f. Dissertação (Mestrado em Saúde Pública) - Escola Nacional de Saúde Pública Sergio Arouca, Fundação Oswaldo Cruz, Rio de Janeiro, 2013.
KUSCHNIR, R.; CHORNY, A. H. Redes de atenção à saúde: contextualizando o debate. Ciênc. saúde colet., Rio de Janeiro, v. 15, n. 5, p. 2307-2316, 2010.

NOVAES, H. M. D. Pesquisa em, sobre e para os serviços de saúde: panorama internacional e questões para a pesquisa em saúde no Brasil. Cad. Saúde Pública, Rio de Janeiro, v. 20, n. 2, p. S147-S173, 2004.

O’DWYER, G. A gestão da atenção às urgências e o protagonismo federal. Ciênc. saúde colet., Rio de Janeiro, v. 15, n. 5, p. 2395-2405, 2010.

PARANÁ. Secretaria de Estado da Saúde. Conselho de Estado da Saúde. Plano Estadual de Saúde Paraná 2012-2015. Curitiba: Secretaria de Estado da Saúde do Paraná, 2013.

RYCHETNIK, L. et al. A glossary for evidence based public health. J Epidemiol Community Health, London v. 5 , n. 7 , p. 538-45, 2004.

SCARPELINI, S. A organização do atendimento às urgências e trauma. Medicina, Ribeirão Preto, Ribeirão Preto, v. 40, n. 3, p. 315-320, 2007.

SILVA, G. S. et al. Redes de atenção às urgências e emergências: pré-avaliação das Unidades de Pronto Atendimento (UPAs) em uma região metropolitana do Brasil. Rev. Bras. Saude Mater. Infant., Recife, v. 12, n.

4, p. 445-458, 2012.

Recebido para publicação em julho de 2015

Versão final em outubro de 2015

Conflito de interesses: inexistente

Suporte financeiro: não houve 